\title{
ÜBER EINIGE FOSSILE PALMENARTIGE HÖLZER AUS DEN OSTLICHEN NIEDERLANDEN
}

\author{
J. VAN DER BURGH UND J. E. MEULENKAMP
}

(Bot. Muscum und Herbarium, Utrecht und Geologisches Institut, Utrecht)

(eingegangen am 25. Februar 1966)

\begin{abstract}
ABSTRAGT
A study was made of the structure of 32 fossil palmlike wood samples, found in diluvial sands in the East of the Netherlands. 27 of them proved to belong to four species of Palmoxylon; two of them are described as new. Five samples belong to a palmroot structure newly described here.
\end{abstract}

\section{Einleitung}

Als im Jahre 1904 Stenzel seine grosse Arbeit über fossile Palmenhölzer veröffentlichte, beschrieb er darin ein Stück Palmenholz aus den Niederlanden als Palmoxylon porosum. Seitdem sind viele sogen. Palmenhölzer in der Umgebung von Eibergen und Vragender gesammelt worden. Es erhob sich die Frage, ob alle diese Hölzer zu Palmoxylon porosum gehören, oder ob sie mehrere Arten darstellen. Die vorliegende Arbeit enthält die Ergebnisse einer Untersuchung von 32 Stücken, wovon $22 \mathrm{zu} P$. porosum Stenzel gehörten.

\section{Material und Methodik}

Die meisten Stücke befinden sich in Privatsammlungen. An dieser Stelle möchten wir den Herren de Graaf, Jansen, Koenderink, Kolstee, Kortenbout van der Sluys, Rhebergen und Stam, die uns ihr Material zur Verfügung stellten recht herzlich danken. Ausserdem verdanken wir noch den Sammlungen des Reichsmuseums für Mineralogie etc, zu Leiden, des Museums Natura Docet zu Denekamp und des Naturhistorischen Museums zu Enschede einige wertvolle Stücke. Die Arbeit wurde ausgeführt im Institut für systematische Botanik der Reichsuniversität Utrecht (Direktor Herr Prof. J. Lanjouw), Abteilung Paläobotanik und Pollenmorphologie (Leitung Herr Prof. F. P. Jonker). Wir danken Herr Roding (1956) für seine Hilfe bei dem Auffinden des Materials und das stätige Interesse mit dem er unsere Arbeit begleitet hat. Wir danken weiter Herrn $H$. A. Elsendoorn für die schnelle Herstellung der benötigten Dünnschliffe und Herrn Frank de Vries für seine unschätzbare Hilfe bei der Mikrofotografie. Ebenfalls danken wir Herrn Dr. K. U. Kramer für das kritische Durchlesen des Manuskriptes.

Von sämmtlichen verkieselten Stücken, die als Diluvialgeschiebe in sandigen Ablagerungen gefunden worden waren, wurden Dünnschliffe angefertigt. Von jedem Stück wurde mindestens ein Querschnitt und wenn nötig, ein Längsschnitt hergestellt. Die verschiedenen Formen wurden zum Bestimmen mit rezenten und fossilen Hölzern verglichen, und zwar mit Dünnschnitten und Beschreibungen. Die Dünnschliffe sind der Sammlung des Instituts für systematische Botanik der Reichsuniversität Utrecht einverleibt worden.

Die Mikrofotografien wurden mit einem Leitz-Ortholux-Mikroskop und mit einer Orthomat-Kamera aufgenommen. 
Für die Nomenklatur wurde die klassische Methode, die der Formgattungen und -arten, beibehalten. Besonders bei "Palmenhölzern" ist grosse Vorsicht geboten, denn viele Monokotyledonen besitzen einen ähnlichen Bau. Dies wurde aufs neue von WEYLAND und KILPPER (1964) unter Beweis gestellt. Sie konnten zeigen, dass Palmoxylon bacillare mindestens teilweise nicht von einer Palme, sondern von einer Bromeliazee stammt.

\section{ERGEBNISSE}

Es fanden sich folgende Hölzer:

\section{ANgIOSPERMAe \\ MONOCOTYLEDONAE}

\section{Palmoxylon Schenk 1882}

\section{Palmoyglon porosum Stenzel 1904 (Tafel I)}

\section{Beschreibung des Holzes}

Dermale Zone: Faserbündel zahlreich, ziemlich dicht beisammen stehend. Das parenchymatische Grundgewebe ist aus isodiametrischen oder etwas gestreckten Zellen zusammengesetzt. Die Interzellularen sind ebenso gross wie die umringenden Zellen und voneinander durch eine etwa vier Zellen dicke Parenchymschicht getrennt. Die Grenze zu der folgenden Zone ist ziemlich scharf.

Subdermale Zone: Hier fehlen die Faserbündel vollkommen. Die Faserleitbündel sind einander bis auf die Hälfte ihrer Breite genähert; ihre Faserteile sind ebenso gross wie die Leitbündelteile oder etwas grösser. Die Faserbelege sind sehr dickwandig. Das parenchymatische Grundgewebe ist aus isodiametrischen oder etwas gestreckten Zellen zusammengesetzt, ebenso wie in der dermalen Zone. Auch hier sind die Interzellularen nur spärlich, aber regelmässig verteilt. Ihre Entfernung ist mehrere Parenchymzellenschichten gross; ihre Grösse ist ungefähr gleich der einer Parenchymzelle.

Zentrale Zone: In der Mitte des Stammes befindet sich die zentrale Zone, welche nach aussen allmählich in die subdermale Zone übergeht. Die Faserleitbündel stehen weiter von einander entfernt, bis zu 2-6 $\times$ ihre Breite. Die Faserteile sind ebenso wie in der subdermalen Zone, sehr dickwandig, aber ihr Umfang ist kleiner als der der Leitbündelteile. Die Leitbündelteile zeigen meist 2, nur vereinzelt 1, 3 oder 4 grosse Gefässe und eine kleine Anzahl von schmalen Elementen. Immer ist das Grundgewebe strahlig um die Faserleitbündel angeordnet. Das Grundgewebe ist ganz locker, die Interzellularen sind von einander durch eine nur eine oder (seltener) zwei Zellen starke Parenchymschicht getrennt; die Parenchymzellen sind nicht isodiametrisch, sondern gestreckt, und mit Ausstülpungen versehen. In diesem $\mathrm{Holz}$ sind die Faserbelege der Faserleitbündel meistens durch röhrenförmige Löcher vertreten. Nach diesem Merkmal hat Stenzel die Art benannt. Er hat sie u.a. auf Grund hiervon dem $P$. germanicum gegenübergestellt, bei dem nicht der Faserteil, sondern der Leitbündelteil des Faserleitbündels zuerst zerstört wird. Das 
Verschwinden gewisser Gewebeteile ist aber ein Erhaltungszustand. Bei Palmoxylon bacillare aus der Braunkohle ist der Leitbündelteil zuerst vermodert (VAN DER BURGH, 1964). $P$. germanicum ist ein verkieselter Stamm (ConwENTz, 1882), ebenso wie dieses und die nachfolgenden Hölzer, von denen auch nur eines ganz vereinzelt ein gutes Leitbündel zeigt. Ferner gibt es unter den untersuchten Stücken solche, bei denen der Faserteil ziemlich gut oder sehr gut erhalten ist, und andere, bei denen man alle Ủbergänge von gutem zu schlechtem Erhaltungszustand finden kann. Dieses gilt nicht nur für $P$. porosum, sondern auch für die drei anderen Arten.

\section{WURzELN}

Im Grundgewebe verschiedener Stücke finden sich in der subdermalen Zone auch Wurzeln. Es sind zweifellos Wurzeln mit Palmenwurzelbau: ein kleiner zentraler Zylinder mit zentralem Xylem, einem Ring von Phloem, einer deutlichen Endodermis und einer dicken Rinde, in der an einer Stelle ein Kranz von Kanälen, wie diese bei den Palmen öfters beobachtet werden, zu finden ist.

Im übrigen gleichen diese Wurzeln sehr den von STOckmans et Willik̀re (1943) als Palmoxylon sp. (Seite 61) beschriebenen Resten. Beim Vergleichen dieses Holzes mit den Beschreibungen schon bekannter Palmoxylonarten können nur die Arten in betracht gezogen werden, die durch das Vorkommen eines lockeren Grundgewebes und das Fehlen der Faserbündel charakterisiert sind. Von diesen schalten die Formen mit nicht strahlig um die Faserleitbündel angeordnetem Grundgewebe aus. Es bleiben nur noch 6 Arten übrig; Palmoxylon porosum, $P$. cellulosum, $P$. blanfordii, $P$. remotum, $P$. fladungii und $P$. rutotii. Von den letzten fünf dieser Hölzer sind in den Faserleitbündeln die Verhältnisse in der Grösse zwischen Faserteil und Leitbündelteil anders als bei diesem Holz. Nur $P$. porosum ist recht ähnlich gebaut, und aus diesem Grunde wird unser Material hier zu dieser Art gestellt.

\section{Palmoxylon pachyoxylon van der Burgh et Meulenkamp (Tafel II)}

\section{Beschreibung des Holzes}

Die Faserleitbündel stehen im Querschnitt regelmässig zerstreut. Ihre Entfernung schwankt zwischen etwa ihrer eigenen Breite in der zentralen Zone und der Hälfte ihrer Breite in der subdermalen Zone. Die Form ist im Querschnitt länglich-rund, der Faserteil ist 2-3 Mal so gross wie der Leitbündelteil. Im Leitbündel findet man meistens 2, zuweilen aber auch 3 oder 4 grosse Gefässe. Das mediane Xylem ist ziemlich gross, ebenso das dickwandige Phloem. Das Leitbündel ist meistens gut erhalten, während der Faserteil oft ganz oder zum Teil geschwunden und teilweise durch Chalzedon ersetzt ist. Die Sklerenchymfasern sind dickwandig, der Faserteil umfasst drei Viertel des Umfanges des Leitbündelteils. Zuweilen findet sich ein zweiter, schmälerer Faserteil gegenüber dem Ersten am Leitbündelteil. Dieser Letztere ist dann amphiphloisch.

Faserbündel fehlen in diesem Holz. 
J. VAN DER BURGH UND J. E. MEULENKAMP: Über einige fossile palmenartige Hölzer aus den östlichen Niederlanden

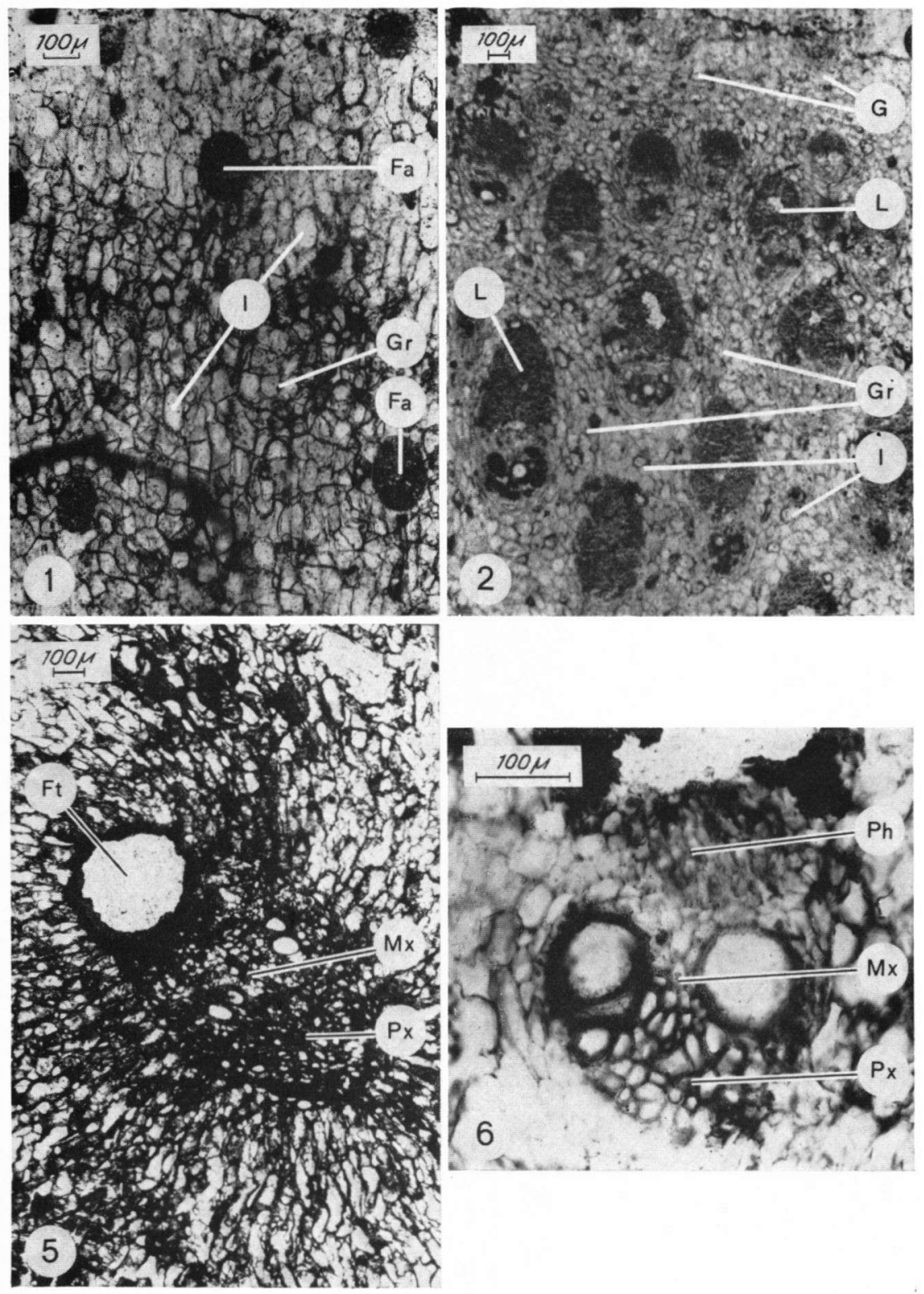

Palmoxylon porosum Stenzel

Fig. 1. Übersicht der dermalen Zone; Fig. 2. Übersicht der subdermalen Zone;

Fig. 3. Übersicht der zentralen Zone; Fig. 4. Faserleitbündel aus der Übergang zwischen zentralen und subdermalen Zone.

TAFEL IA 

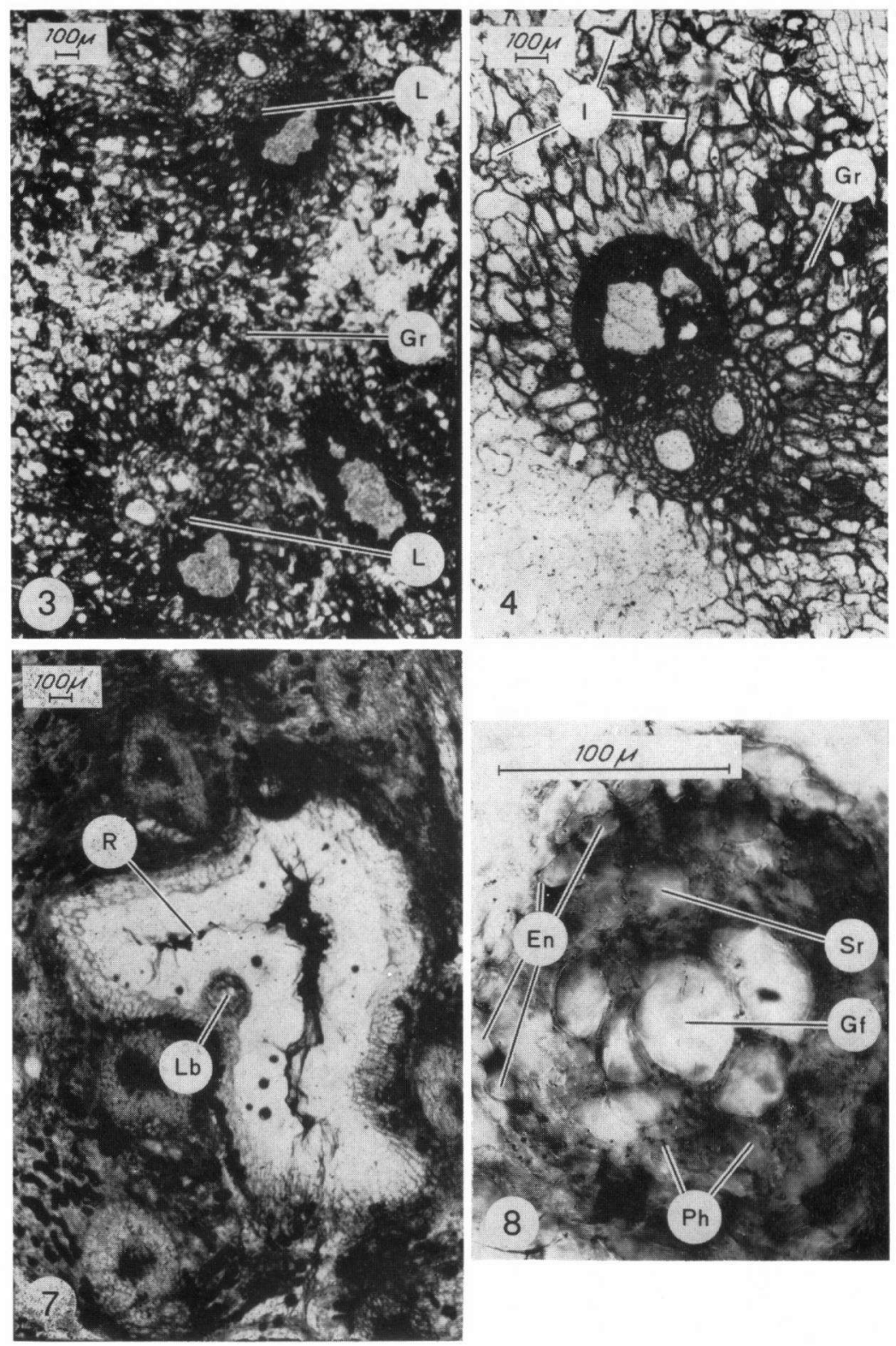

Palmoxylon porosum StenzelY(Fortsetzung)

Fig. 5. Faserleitbündel aus der zentralen Zone; Fig. 6. Leitbündelteil eines Faserleitbündels; Fig. 7. Übersicht der subdermalen Zone mit einem Wurzelspur; Fig. 8. Leitbündel der Wurzel aus Fig. 7 . 

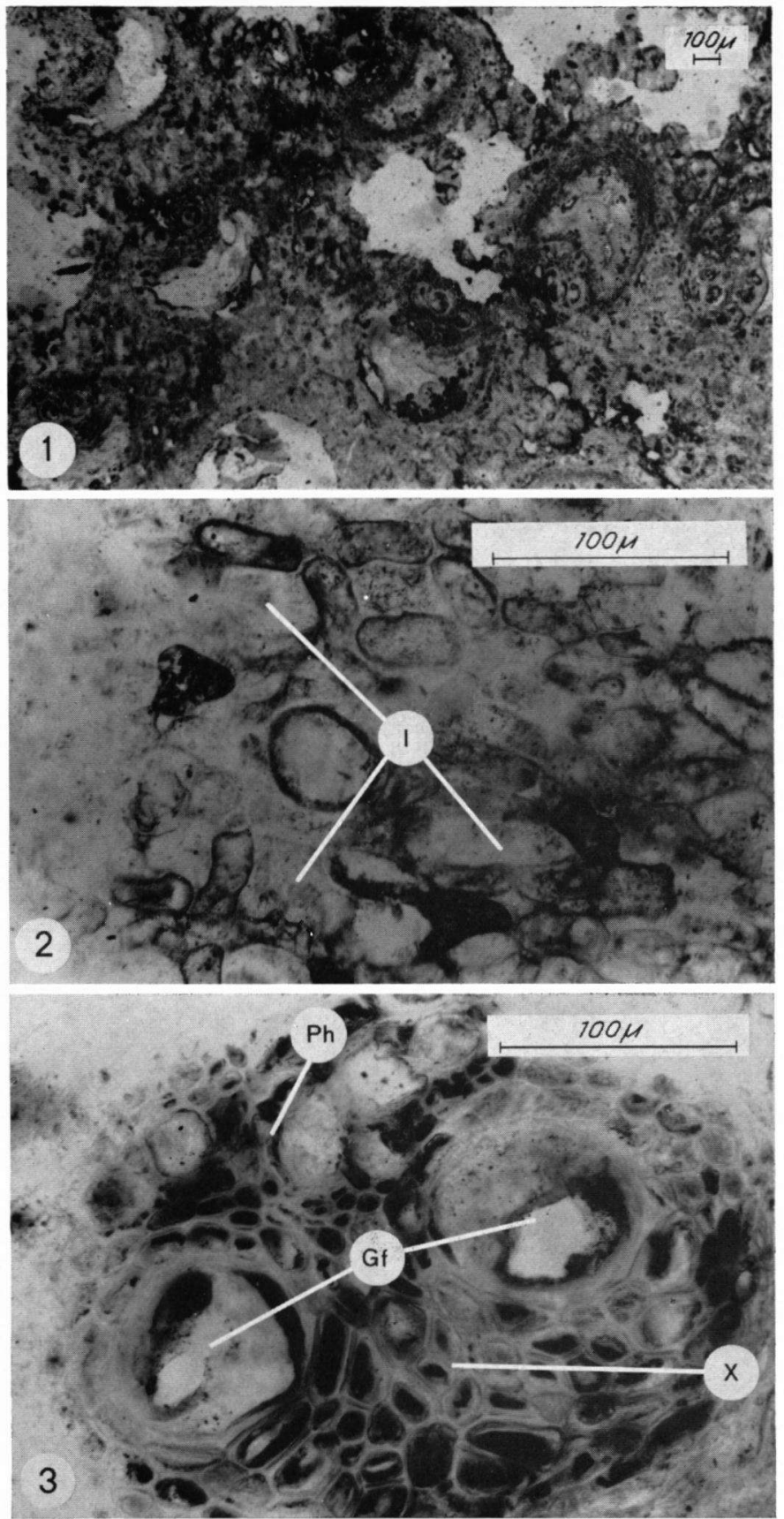

Palmoxylon pachyoxylon van der Burgh et Meulenkamp

Fig. 1. Ubersicht; Fig. 2. Grundgewebe; Fig. 3. Leitbündelteil des Faserleitbündels.

TAFEL II 

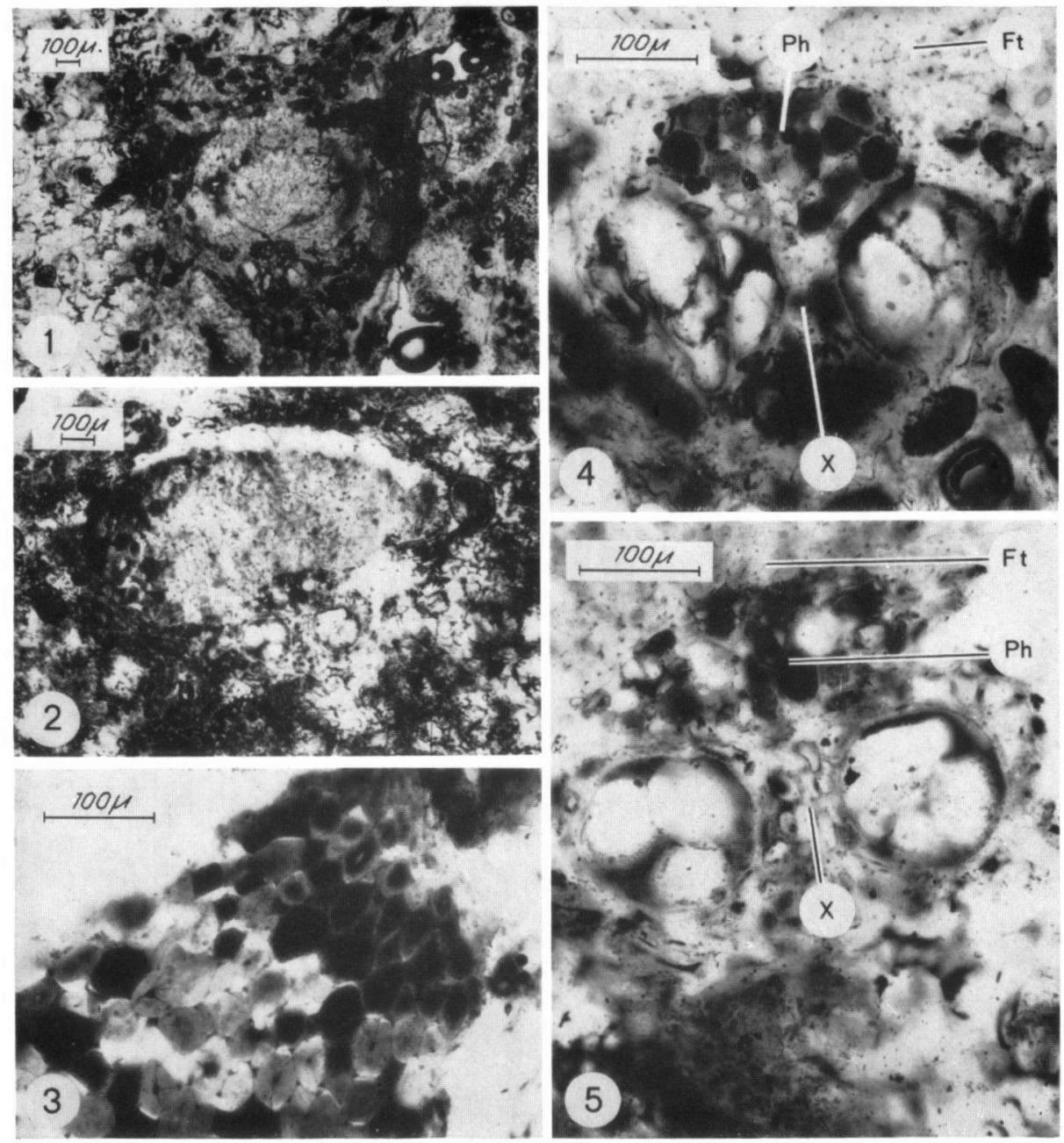

Palmoxylon variabile Vater

Fig. 1. Faserleitbündel im Grundgewebe; Fig. 2. Faserleitbündel; Fig. 3. Faserteil eines Faserleitbündels; Fig. 4. Leitbündelteil des Bündels aus Fig. 1; Fig. 5. Leitbündeiteil des Bündels aus Fig. 2. 

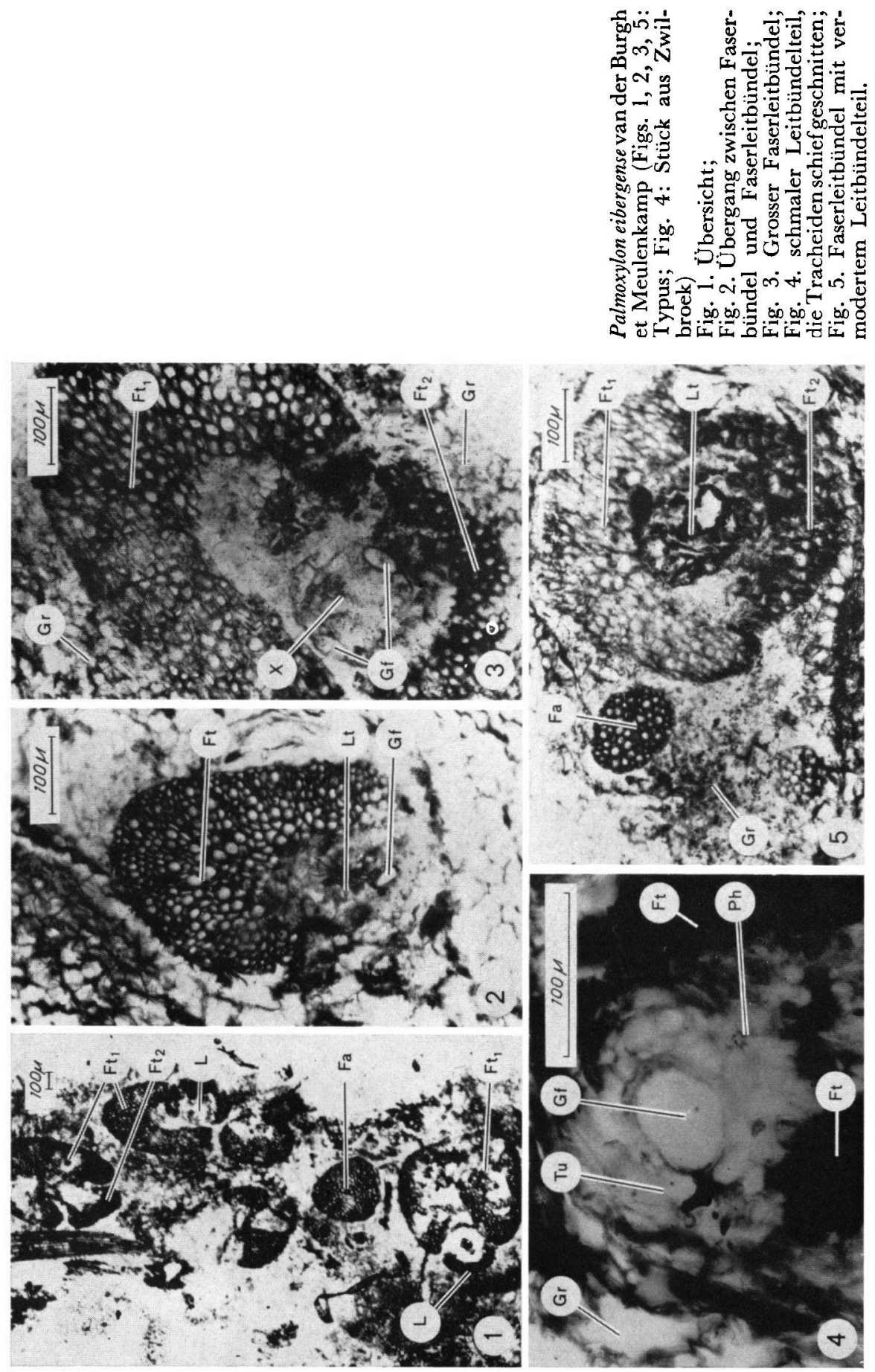

TAFEL IV 


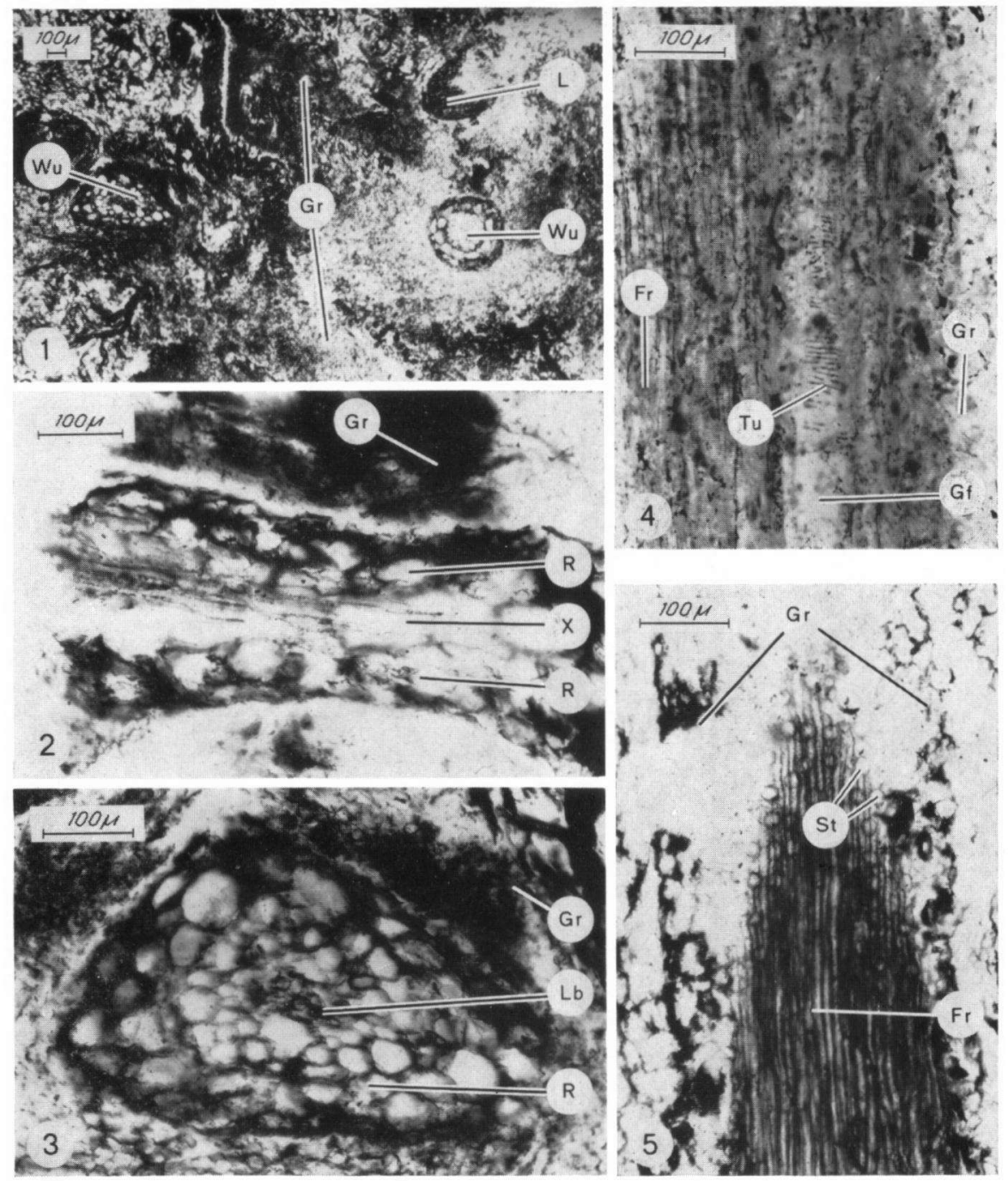

Palmoxylon eibergense van der Burgh et Meulenkamp (Figs. 1-4: Typus; Fig. 5: Stück aus Zwilbroek)

Fig. 1. Übersicht mit Wurzeln; Fig. 2. Längsschnitt einer Wurzel; Fig. 3. Wurzelquerschnitt; Fig. 4. Längsschnitt eines Faserleitbündels; Fig. 5. Längsschnitt des Faserteils eines Faserleitbündes. 

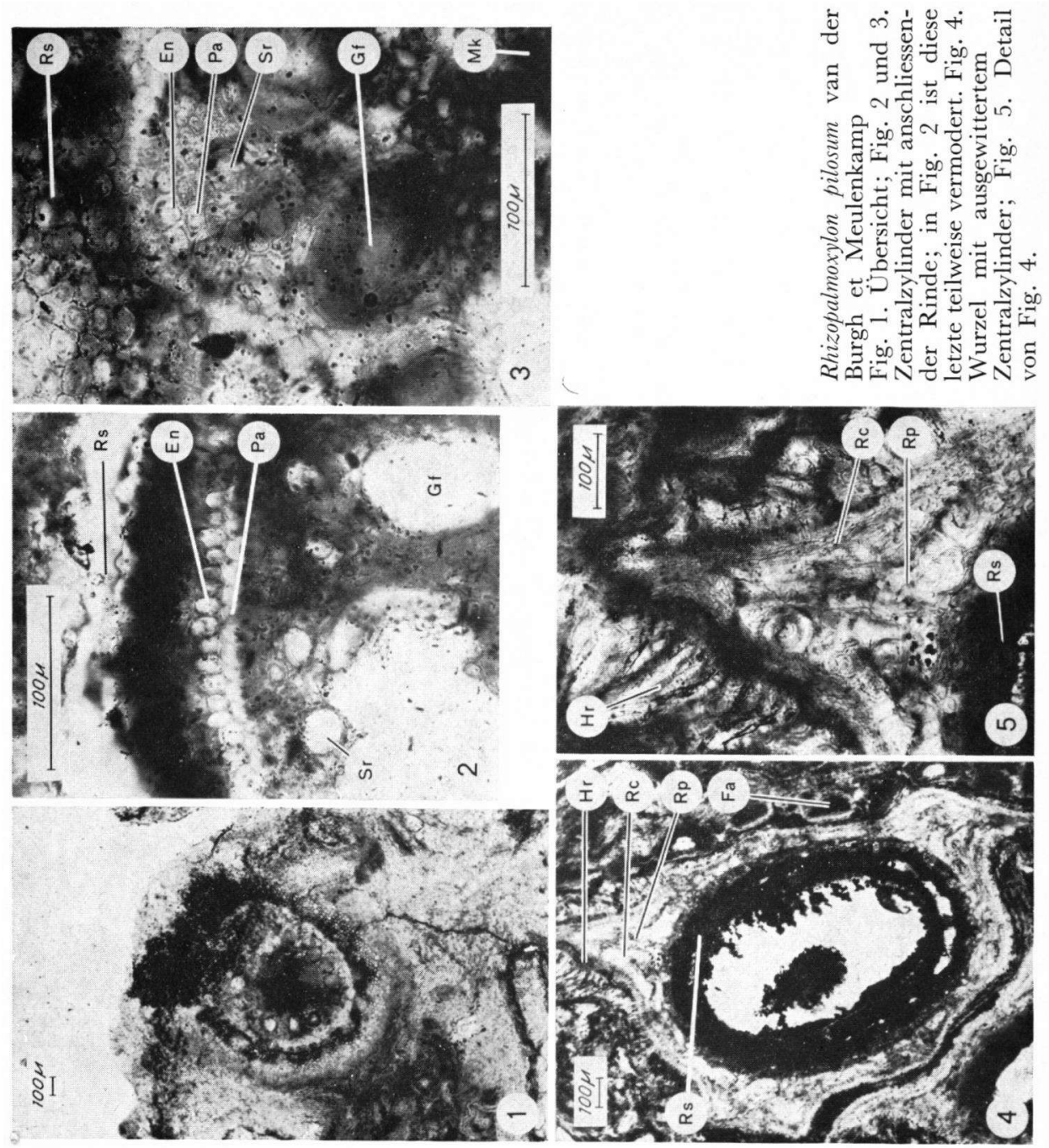


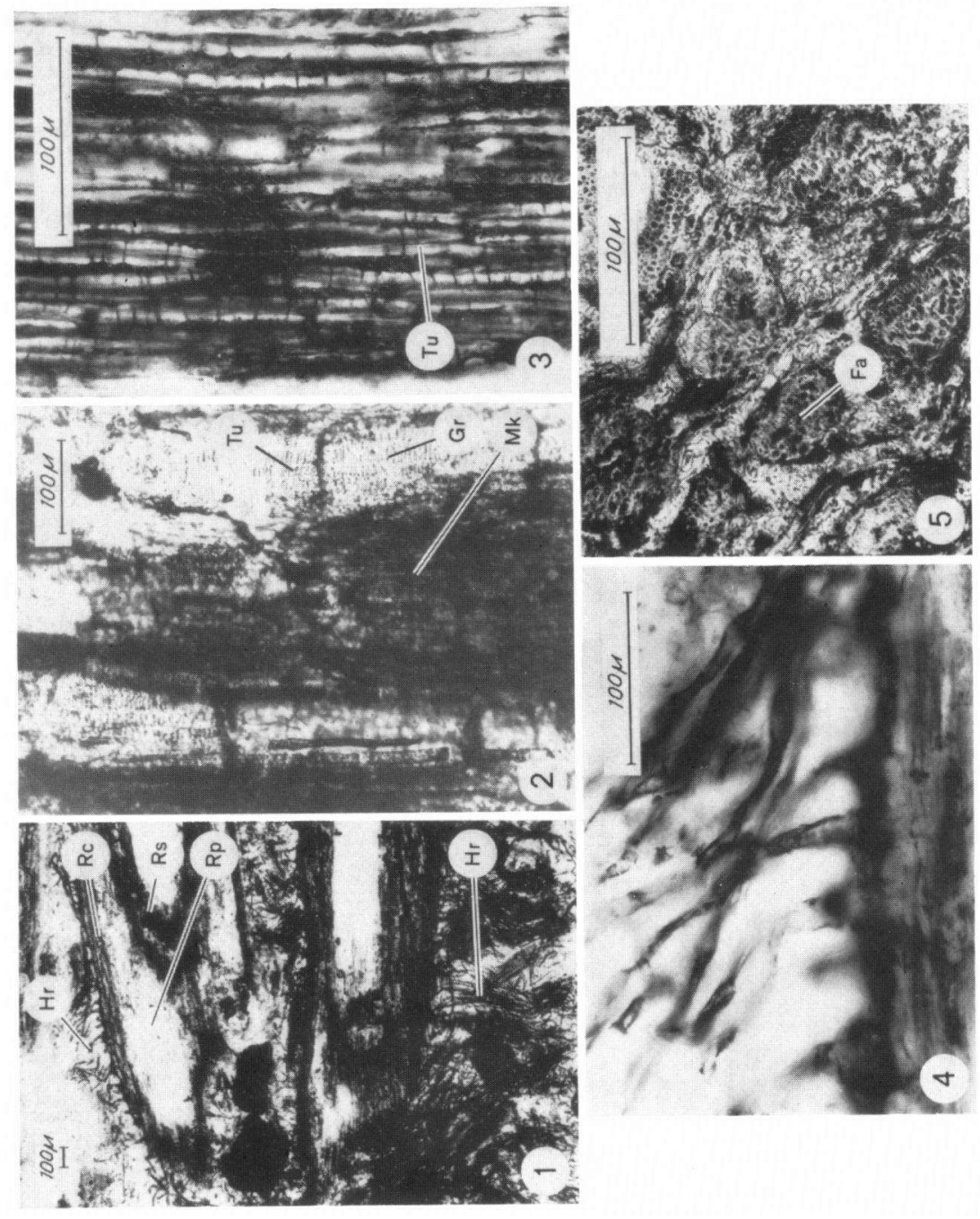

Rhizopalmoxylon pilosum van der Burgh et Meulenkamp

Fig 1. Übersicht einer Längsschnitt; Fig. 2. Längsschnitt eines Zentralzylinders; Fig. 3. Längsschnitt der Sklerenchymatischen Rinde; Fig. 4. Haare; Fig. 5. Reste der Stammrinde (Cortex). 
Das Grundgewebe ist locker, mit vielen grossen Interzellularen und parenchymatischen, unregelmässig geformten Zellen. Um die Faserleitbündel bildet das Grundgewebe eine bis zu 2 oder 3 Zellen starke Schicht; darauf folgt das lockere Parenchym mit den vielen Interzellularen. Diese Parenchymzellen stehen strahlig rundum die Faserleitbündel angeordnet, doch ist das nicht besonders ausgeprägt. Im Längsschnitt fällt sogleich der grosse Holzteil auf, dessen Treppentüpfel schön konserviert sind. An die Faserbelegen sind viele Stegmata ausgebildet.

Von dieser Art lag nur ein Stück vor: ein kleines Stück aus der Sammlung des Reichsmuseums für Mineralogie etc. zu Leiden (Nr 62321), dies ist zugleich der Typus. Als Fundort wird Sibculo angegeben. Schon am ganzen Stück kann man sehen, dass die Faserleitbündel schmäler sind und mehr gedrängt stehen als bei der vorigen Art. Es stammt aus der subdermalen und zentralen Schicht eines Stammes.

Im Bau des Faserteils der Faserleitbündel unterscheidet sich dieser Art sofort von $P$. porosum. Auch der Bau der Leitbündel ist anders. Vergleicht man dieses Holz mit Palmenhölzern ohne Faserbündel und mit lockerem Parenchym, so ergibt sich, dass keine der oben bei $P$. porosum genannten Arten mit diesem Holz in genügend Einzelheiten übereinstimmt um auf Zusammengehörigkeit schliessen zu können. Auch $P$. paniselianum hat abweichende Merkmale. Besonders das Grundgewebe ist verschieden (Stockmans et Wirlitere, 1943). Es handelt sich hier um eine neue Art, die nach dem verhältnismässig starken Holzgewebe Palmoxylon pachyoxylon genannt wird. Die Beschreibung soll als Diagnose gelten.

\section{Palmoxylon variabile Vater 1884 (Tafel III)}

\section{Beschreibung des Holzes}

Die Faserleitbündel sind breit, ihr Faserteil ist um ein Mehrfaches grösser als ihr Leitbündelteil, der von dem Faserteil nur wenig oder gar nicht umfasst wird. Die Fasern sind sehr starkwandig. Das Leitbündel enthält ein medianes kleines Protoxylem und einige Metaxylemelemente. Zu beiden Seiten steht meist nur ein grosses Gefäss. An der Sklerenchymkappe findet man einen Phloemstrang mit dünnwandigen Elementen. Auch hier ist in der Regel der Leitbündelteil besser erhalten als der Faserteil. Faserbündel fehlen. Das Grundgewebe ist parenchymatisch, dicht und aus ziemlich regelmässigen Zellen gebildet.

Auch von dieser Art liegt nur ein Stück vor. Es ist ein schmales, brettförmiges Stück, dass auf Grund der teilweise dicht gedrängten Stellung der Faserleitbündel und ihrer mehr oder weniger deutlichen Orientierung wohl der subdermalen Zone angehört. Als Fundort wird Westerhaar angegeben. Es stammt aus die Sammlung von Herrn Rhebergen zu Schonebeek. Von den Palmenhölzern mit dichtem Grundgewebe und ohne Faserbündel gleicht unser $\mathrm{Holz}$ den $\mathrm{Be}-$ schreibungen und Abbildungen von $P$. germanicum, $P$. loppemense und 
$P$. variabile am meisten. $P$. loppemense hat aber viel strahlig angeordnetes Grundgewebe, $P$. germanicum ein dichtes, aus isodiametrischen Zellen zusammengesetztes Grundgewebe mit nur kleinen Interzellularen, aber auch hier sind die Grundgewebezellen um die Übergangsbündel herum strahlig angeordnet, was bei unserem Stück nicht der Fall ist. $P$. variabile dagegen zeigt nur etwas verlängerte, den Faserbündeln anliegende Grundgewebezellen. Andere Punkte der Übereinstimmung sind: der gegenseitige Abstand der Faserleitbündel (schwankend von weniger bis etwas mehr als ihr Durchmesser), ihre sehr mannigfaltige Form, und der Faserteil, der an Grösse den Leitbündelteil stets übertrifft und auch in der Form ganz mit den Beschreibungen und Abbildungen von Vater (1884) und Stenzel (1904) übereinstimmt. Aus diesem Grunde wird unser Holz zu Palmoxylon variabile gestellt.

Palmoxylon eibergense van der Burgh et Meulenkamp (Tafel IV, V)

\section{Beschreibung des Holzes}

Die Bündel zeigen sich schon am Stück recht verschieden von denen der vorigen Arten, indem sie stark wellig verlaufen. Im parenchymatischen Grundgewebe, das aus isodiametrischen oder etwas gestreckten Zellen zusammengesetzt ist, findet man sowohl Faserbündel als auch Faserleitbündel. Ausserdem gibt es alle möglichen Úbergänge zwischen beiden Formen von Bündeln. Schliesslich finden sich ziemlich viele Wurzelreste. Die Faserleitbündel stehen in allen Zonen des Zentralzylinders gleich dicht gedrängt. Im Inneren des Stammes zeigen sie keine Orientierung. Die Faserbelege sind dünnwandig und ziemlich oft zweiteilig, d.h. es ist ein grosses Faserbündel vorhanden, wie bei fast allen Palmoxyla, und ein zweites, kleineres am gegenüberliegenden Teil des Leitbündels. Oft sind beide Faserbündelteile verbunden und umschliessen zusammen den ganzen Leitbündelteil. Der Leitbündelteil enthält 1-5 ziemlich enge Gefässe. Die Übergänge zum Faserbündel werden gebildet durch Bündel mit einem verhältnismässig grossen Faserteil und einem kleinen oder sogar ganz winzigen Leitbündelteil.

\section{WURzELN}

Neben den Faserleitbündeln und Faserbündeln finden sich in allen Stücken dieser Art noch Wurzeln, deren Erhältungszustand mit dem des Faserleitbündels vergleichbar ist. Sie zeigen den gewöhnlichen Bau: dicke Rinde, bis zu 4 Zellen dick, also weniger als die der bisher beschriebenen Palmenwurzel, und nicht in sklerenchymatische und parenchymatische Gewebeschichten differenziert, sondern rein Parenchymatisch, also verschieden von den uns bekannten Palmenwurzeln. Der Zentralzylinder ist ausserordentlich schmal und umfasst nur wenige Xylem- und Phloemelemente.

Von dieser Art liegen drei Stücke vor: ein Stück aus Zwolle bei Groenlo (Sammlung Stam) ein zweites und ein drittes aus Zwilbroek bei Eibergen (Sammlungen Koenderink, Bilthoven und Naturhistorisches Museum, Enschede). Das erste Stück stammt aus der 
subdermalen und zentralen Zone des Stammes, das zweite hochstwahrscheinlich nur aus der subdermalen Zone, und das dritte ist zu klein, als dass mit genügender Sicherkeit etwas darüber ausgesagt werden könnte. Als Typus wird das Stück aus Zwolle bei Groenlo gewählt. Es gibt viele Palmenhölzer mit dichtem Grundgewebe und Faserbündeln neben den Faserleitbündeln. Von allen diesen gibt es aber nur wenige mit doppelten Faserbündeln an den Faserleitbündeln: $P$. parvifasciculosum, $P$. scleroticum, $P$. hillebrandtii, $P$. wentii und $P$. pietzschii. Keine dieser Arten stimmt aber völlig mit unserem Holz überein, denn bei $P$. parvifasciculosum ist der Faserteil schmaler als der Leitbündelteil, während bei unserem Holz die Verhältnisse gerade umgekehrt sind. Bei $P$. scleroticum sind die Grundgewebezellen angeblich zum Teil verholzt (VATER, 1884) was bei unserem Holz nicht der Fall ist. Auch sind dort, ebenso wie bei $P$. wentii, $P$. hillebrandtii und $P$. pietzschii, die Sklerenchymfasern stark verdickt (Stockmans et Willière, 1943; Pax und Gingelsheim in PAx, 1907; Schönfeld, 1955). Bei unserer Holzprobe dagegen sind sie verhältnismässig dünnwandig. Beim Durchsehen der Literatur stiessen wir auf die Beschreibungen von $P$. bacillare bei JuRAsKY (1930) und bei VAN DER BURGH (1964) und von Bromeliaceophyllum rhenanum bei Weyland und KilpPER (1964). Besonders mit der Beschreibung und den Figuren der letzteren Autoren besteht gute Übereinstimmung. Es finden sich in beiden Hölzern Utbergänge zwischen Faserbündeln und Faserleitbündeln, eine gleichmässige Verteilung der Bündel über den ganzen Stammdurchschnitt, und nicht immer stark sklerotisierte Sklerenchymkappen. Hinzu kommt noch der abweichende Bau der Wurzeln. Aus diesem Grunde liegt es nahe, das Holz systematisch in der Nähe von Bromeliaceophyllum rhenanum zu stellen. Es bestehen auch gewisse Unterschiede zwischen unserem Holz und Bromeliaceophyllum rhenanum: eine Zweiteilige Sklerenchymkappe findet sich nur in unserem $\mathrm{Holz}$, und Bromeliaceophyllum rhenanum zeigt in den äusseren Schichten stark verdickte Sklerenchymfasern, während bei unserem Holz alle Sklerenchymfasern gleich stark sind.

Uber die Pflanze, zu der dieser Stamm gehört, wissen wir gar nichts, und es erscheint darum angebracht, das Holz in die Gattung Palmoxylon unter dem Namen Palmoxylon eibergense einzureihen. Als Diagnose soll die Beschreibung gelten.

Rhizopalmoxylon Gothan 1942.

Rhizopalmoxylon pilosum van der Burgh et Meulenkamp

Beschreibung des Holzes

(Tafel VI, VII)

Dieses "Holz" ist aus zahlreichen Wurzeln, die mit einander verflochten sind, zusammengesetzt. Ausserdem finden sich noch Reste der dermalen Zone eines Palmenstammes.

Im Innern einer Wurzel befindet sich in der Mitte das sklerenchymatische Mark, ringsum vom Xylem umschlossen. Darauf folgt ein Ring von Phloem, nach aussen abgeschlossen von einer nur eine 
Zelle starken Parenchymschicht. Der zentrale Zylinder wird nach aussen begrenzt von einer Endodermis, die etwas starkwandiger ist als die Phloem- und Parenchymschichten. Es folgt dann die Rinde, welche sehr dick ist. Sie ist von innen nach aussen folgendermassen strukturiert: zuerst sklerenchymatisch, dann parenchymatisch, und zuletzt kollenchymatisch. Die Epidermis trägt viele haarähnliche Papillen.

Oft ist der Zentralzylinder ausgewittert. In der sklerenchymatischen Rinde ist die Mittelpartie vermodert und nicht durch Quarz ersetzt worden, und der runde Zentralzylinder fällt als kleines Stäbchen heraus. Zwischen den Wurzel findet man vereinzelt kleine Gruppen von Stammrindengewebe, deren Grundgewebe ganz zerdrückt ist. Die Wurzelrinde wirft oft sehr starke Falten. Im Längsschnitt kann man die Treppentüpfel der Holzgefässe beobachten. Auch ist der Bau der Rinde im Längsschnitt oft besser zu unterscheiden. Die Sklerenchymatische innere Kortex zeigt sehr schöne Tüpfel, genau wie sie z.B. auch bei Hyospathe elegans wahrzunehmen sind.

Das Material kann aus Grund des palmenwurzelähnlichen Baues zu Rhizopalmoxylon Gothas (1942) gestellt werden. Wegen des verschiedenen Baues des zentralen Zylinders (nur ein Ring von Gefässen) wird es hier als einer neuen Art beschrieben unter dem Namen Rhizopalmoxylon pilosum.

Der Artnahme wurde gewählt auf Grund der vielen Epidermispapillen, die wie Haare aussehen und die Wurzeln ganz dicht verflechten.

Als Diagnose soll die Beschreibung gelten.

Von dieser Art liegen 5 Stücke vor. Vier davon sind so stark gebleicht und waren vor dem eintreten der Verkieselung auch so stark vermodert, dass nicht viel Struktur daran zu beobachten ist. Das Fünfte war besser erhalten und gestattete die mikroskopischen Strukturen zu untersuchen. Dieses letztes Stück wurde zum Typus gewählt und stammt aus der Sammlung von Herrn de Graaf, Arnhem, Fundort Rosendaal. Die vier andere Stücke stammen aus der Umgebung von Borne und Almelo.

\section{ZUSAMMENFASSUNG}

Es wurden insgesamt 32 Geschiebehölzer palmenartiger Natur aus den östlichen Niederlanden (Provinzen Gelderland und Overijssel) bearbeitet. Es stellte sich heraus, dass 27 Stücke zu 4 Palmoxylon-Arten gehören, darunter 2 neue; 5 Stücke gehören zu einer neu beschriebenen Palmenwurzelstruktur.

\section{ERKLÅRUNG DER BUCHSTABEN IN DEN FIGUREN}

En: Endodermis

Fa: Faserbündel

Fr: Faser

Ft: Faserteil

G: Grenze der dermalen Zone

Gf: Gefäss

Gr: Grundgewebe

Hr: Haare

I: Interzellular

L: Faserleitbündel
Lb: Leitbündel

Lt: Leitbündelteil des Faserleitbündels

Mk: Mark

Mx: Metaxylem

Pa: Parenchym

Ph: Phloem

Px: Protoxylem

R: Rinde

Rc: kollenchymatische Rinde

Rp: parenchymatische Rinde 
UBER EINIGE fOSSILE PALMENARTIGE HÖLzER AUS DEN OSTLICHEN NIEDERIANDEN 283

Rs: sklerenchymatische Rinde

Sr: Siebrohr

St: Stegmata
Tu: Tüpfel

X: Xylem

Wu: Wurzel

\section{LITERATUR}

Burgh, J. VAN DER. 1964. Acta Bot. Neerl. 13: 250.

Conwentz, H. 1882. Jahrb. Kgl. Preuss. Geol. Landesamt für 1881, Beiheft: 144.

Gothan, W. 1942. Zeitschr. f. Geschiebeforsch. u. Flachlandgeol. 18.

Jurasky, K. A. 1930. Braunkohle 29 Heft 51: 117.

Pax, F. 1907. Engler, Bot. Jahrb. 38: 272.

Roding, G. M. 1956. Grondboor en Hamer No. 3.

Sahönfeld, E. 1955. Palaeontografica 99 Abt. B: 1.

Stenzel, K. G. 1904. Beitr. Geol. Osterr. Ung. 16: 107.

Stockmans, F. et Y. Willière. 1943. Mém. Mus, roy. hist. nat. Belg. 100.

VAter, H. 1884. Zeitschr. Deutsch. Geol. Ges. 36: 783.

Weyland H. et K. Krlpper. 1964. Palaeontografica 115 Abt. B: 1. 